Groups Geom. Dyn. 4 (2010), 251-261

DOI $10.4171 / \mathrm{GGD} / 82$
Groups, Geometry, and Dynamics

(C) European Mathematical Society

\title{
Sigma invariants of direct products of groups
}

\author{
Robert Bieri and Ross Geoghegan*
}

\begin{abstract}
The Product Conjecture for the homological Bieri-Neumann-Strebel-Renz invariants is proved over a field. Under certain hypotheses the Product Conjecture is shown to also hold over $\mathbb{Z}$, even though D. Schütz has recently shown that the Conjecture is false in general over $\mathbb{Z}$. Our version over $\mathbb{Z}$ is applied in a joint paper with D. Kochloukova [5] to show that for all $n$ Thompson's group $F$ contains subgroups of type $F_{n}$ which are not of type $\mathrm{FP}_{n+1}$.
\end{abstract}

Mathematics Subject Classification (2010). 20E06, 20F65, 55U25.

Keywords. Bieri-Neumann-Strebel invariants, product conjecture, products of groups.

\section{Introduction}

Let $G$ be a group. A (real additive) character on $G$ is a homomorphism $\chi: G \rightarrow \mathbb{R}$ from $G$ to the additive group of real numbers. Two non-zero characters $\chi, \chi^{\prime}: G \rightarrow \mathbb{R}$ are equivalent if they differ by a positive multiple, i.e., $\chi^{\prime}=r \chi$ for some $r>0$. The equivalence class of $\chi$ is denoted by $[\chi]$. The equivalence class of a non-zero character $\chi$ should be thought of as the straight open ray from 0 through $\chi$ in the real vector space $\operatorname{Hom}(G, \mathbb{R})$ of all characters. The dimension of this vector space is the torsion-free rank of the abelianization $G / G^{\prime}$ of $G$. Thus, when $G / G^{\prime}$ is finitely generated, the set of equivalence classes of non-zero characters, denoted by $S(G)$, is a geometric sphere on which we can do spherical geometry.

Our ground ring $R$ is assumed to be a domain, i.e., a commutative ring with $1 \neq 0$ which is without zero divisors. When we consider $R$ as an $R G$-module, we always refer to the trivial action of $G$ on $R$. Each $[\chi]$ defines a submonoid $G_{\chi}:=\{g \in G \mid \chi(g) \geq 0\}$, and $R$ will also be considered as a trivial $R G_{\chi}$-module. In the paper [7] of Bieri and Renz ${ }^{1}$ the Sigma-invariants (or geometric invariants) are defined for each integer $n \geq 0$ by

$$
\Sigma^{n}(G ; R):=\left\{[\chi] \in S(G) \mid R \text { is of type } \mathrm{FP}_{n} \text { over } R G_{\chi}\right\} .
$$

\footnotetext{
* This work was partially supported by a grant from the Deutsche Forschungsgemeinschaft.

${ }^{1}$ In [7] things are done over the ground ring $\mathbb{Z}$, but everything in that paper goes through over $R$. In our application to Thompson's Group in [5] we will need to compare the situation over $\mathbb{Z}$ with that over $\mathbb{Q}$.
} 
The basic reference for these is [7]. A less economical but more intuitive definition is given in Section 3. While it is clear that $\Sigma^{0}(G ; R)=S(G)$ the case $n=0$ will play a role in what follows.

There are also homotopical versions of these invariants, denoted $\Sigma^{n}(G)$, which were introduced in [7] ${ }^{2}$, Remark 6.1. The relationship between $\Sigma^{n}(G ; R)$ and $\Sigma^{n}(G)$ is the usual relationship in topology between homology with $R$-coefficients and homotopy; see Theorem 3.2.

This paper is about the behavior of the $\Sigma$-invariants with respect to direct products of groups. Consider two groups $G$ and $H$. The vector spaces $\operatorname{Hom}(G \times H, \mathbb{R})$ and $\operatorname{Hom}(G, \mathbb{R}) \oplus \operatorname{Hom}(H, \mathbb{R})$ are identified in the usual way. This embeds the spheres $S(G)$ and $S(H)$ canonically as subspheres of $S(G \times H)$ so that when $\chi \in \operatorname{Hom}(G, \mathbb{R})$ and $\chi^{\prime} \in \operatorname{Hom}(H, \mathbb{R})$ the notations $\chi+\chi^{\prime}$ and $\left(\chi, \chi^{\prime}\right)$ both describe the character $(g, h) \mapsto \chi(g)+\chi^{\prime}(h)$. Thus, as the non-zero characters $\chi$ and $\chi^{\prime}$ vary in their respective rays $[\chi]$ and $\left[\chi^{\prime}\right]$, the point $\left[\chi+\chi^{\prime}\right] \in S(G \times H)$ varies among the points of the open spherical geodesic whose end points are $[\chi]$ and $\left[\chi^{\prime}\right]$. With the usual interpretation of $S(G \times H)$ as the join of $S(G)$ and $S(H)$, this spherical geodesic is the join-segment from $[\chi]$ to $\left[\chi^{\prime}\right]$. Thus, when $P \subseteq S(G)$ and $Q \subseteq S(H)$ their join is

$$
P * Q:=\left\{\left[\chi+\chi^{\prime}\right] \mid[\chi] \in P,\left[\chi^{\prime}\right] \in Q\right\} \cup P \cup Q
$$

It is sometimes convenient to extend the notations $\chi+\chi^{\prime}$ and $\left(\chi, \chi^{\prime}\right)$ to include the possibility $\chi=0$ or $\chi^{\prime}=0$ thus collecting the two endpoints of the geodesic in the same notation. (The reason is that when $\chi^{\prime}=0$ and $\chi \neq 0$, or vice versa, $\chi+\chi^{\prime}$ is a non-zero character on $G \times H$.)

Recall that the group $G$ has type $\operatorname{FP}_{n}(R)$ if there is a free $R G$-resolution of $R$ which is finitely generated in dimensions $\leq n$; when $R=\mathbb{Z}$ one simply says that $G$ has type $\mathrm{FP}_{n}$. When we discuss $\Sigma^{n}(G ; R)$, we will always assume that the group in question has type $\operatorname{FP}_{n}(R)$. Similarly, $\Sigma^{n}(G)$ is only defined when $G$ has the topological finiteness type ${ }^{3} F_{n}$.

The question of a formula for the homotopical $\Sigma$-invariants of direct products of groups is reduced to the corresponding question for the homological invariants by the following:

\section{Theorem 1.1.}

$$
\Sigma^{n}(G \times H)=\left(\Sigma^{n}(G \times H ; \mathbb{Z})-(S(G) \cup S(H))\right) \cup\left(\Sigma^{n}(G) \cup \Sigma^{n}(H)\right) .
$$

This first appeared in [13]; see [2], Proposition 4.6, for a proof.

\footnotetext{
${ }^{2}$ In [7] these are denoted by ${ }^{*} \Sigma^{n}(G)$. Again, we have $\Sigma^{0}(G)=S(G)$. The case $n=1$ is a recasting of the Bieri-Neumann-Strebel Invariant introduced earlier in [6].

${ }^{3} G$ has type $F_{n}$ if there is a $K(G, 1)$-complex with finite $n$-skeleton. For details on finiteness properties of groups see for example [11].
} 
We denote the complement of any subset $A$ of a sphere by $A^{c}$. The Direct Product Formula (whether true or false - that is the subject of this paper) reads as follows:

$$
\Sigma^{n}(G \times H ; R)^{c}=\bigcup_{p=0}^{n} \Sigma^{p}(G ; R)^{c} * \Sigma^{n-p}(H ; R)^{c} .
$$

The $\subseteq$ inclusion of this statement is a theorem due to H. Meinert:

Theorem 1.2 (Meinert's inequality).

and

$$
\Sigma^{n}(G \times H ; R)^{c} \subseteq \bigcup_{p=0}^{n} \Sigma^{p}(G ; R)^{c} * \Sigma^{n-p}(H ; R)^{c}
$$

$$
\Sigma^{n}(G \times H)^{c} \subseteq \bigcup_{p=0}^{n} \Sigma^{p}(G)^{c} * \Sigma^{n-p}(H)^{c} .
$$

Meinert did not publish this, but a proof can be found in [10], Section 9. The paper [2] also contains a proof of the homotopy version ${ }^{4}$.

In this paper we consider the $\supseteq$ inclusion of the Direct Product Formula. Here, caution is needed as there are counterexamples. A counterexample to the homotopy version was given by Meier, Meinert and vanWyk in [12], Section 6. Recently, a counterexample to the homological version has been given by D. Schütz in [15] for the case where $R=\mathbb{Z}$. This involves the product of two right-angled Artin groups. (The article [2] contains the incorrect statement - for which the first author owes an apology - that the computation of $\Sigma^{n}(G, \mathbb{Z})$ for right angled Artin groups $G$ given in [12] - see also [8] - establishes the $\supseteq$ direction of the Direct Product Formula for those groups when $R=\mathbb{Z}$.)

In this paper we prove:

Theorem 1.3. When $R$ is a field, the Direct Product Formula is true.

As a corollary we have:

Theorem 1.4. When $\Sigma^{p}(G ; \mathbb{Z})=\Sigma^{p}(G ; \mathbb{Q})$ and $\Sigma^{p}(H ; \mathbb{Z})=\Sigma^{p}(H ; \mathbb{Q})$ for all $p \leq n$ then the $\mathbb{Z}$-version of the Direct Product Formula is true; i.e.,

$$
\Sigma^{n}(G \times H ; \mathbb{Z})^{c}=\bigcup_{p=0}^{n} \Sigma^{p}(G ; \mathbb{Z})^{c} * \Sigma^{n-p}(H ; \mathbb{Z})^{c} .
$$

Proof. This follows from Meinert's Inequality (Theorem 1.2) together with the fact that one always has: $\Sigma^{p}(\cdot ; \mathbb{Z}) \subseteq \Sigma^{p}(\cdot ; \mathbb{Q})$.

\footnotetext{
${ }^{4}$ That our statement of Meinert's Inequality is equivalent to the statement in [10] requires a little work. The case where one of the characters is zero is covered by our Proposition 5.1. The other case is a straightforward exercise.
} 
Theorem 1.4 is applied in [5] to get information about subgroups of Thompson's Group $F$. There the $\Sigma$-invariants of $F$ are computed, and one has $\Sigma^{p}(F ; \mathbb{Z})=$ $\Sigma^{p}(F ; \mathbb{Q})$ for all $p$, so that, by Theorem 1.4 , the $\mathbb{Z}$ version of the Direct Product Formula is true with $G=H=F$. A consequence is that, for all $n, F$ contains subgroups of type $F_{n}$ which are not of type $\mathrm{FP}_{n+1}$

A corollary of the proof of Theorem 1.3, also proved by Schütz in [15], is:

Theorem 1.5. The Direct Product Formula is true when $R=\mathbb{Z}$ provided that $n \leq 3$.

This is explained in Remark 5.3.

We wish to acknowledge the roots of the present paper. Computing $\Sigma^{n}(G \times H ; \mathbb{Z})$ was a much discussed theme in Frankfurt around 1990, and in this paper we take full advantage of those discussions, more than we are able to track down in the literature. We can certainly refer to Holger Meinert's diploma thesis [14] which contains the $\mathbb{Z}$-version of his inequality, stated above in Theorem 1.2. We can also refer to Ralf Gehrke's doctoral thesis [9] and [10] which implies equality in the $\mathbb{Z}$-version in some special cases. The survey article [2] contains more details, including a proof of Gehrke's result.

\section{Valuations extending a character}

Let $\chi: G \rightarrow \mathbb{R}$ be a character on $G$. If $M$ is an $R G$-module, a valuation on $M$ extending $\chi$ is a function $v: M \rightarrow \mathbb{R} \cup\{\infty\}$ satisfying the axioms

$$
\begin{aligned}
v\left(m+m^{\prime}\right) & \geq \min \left\{v(m), v\left(m^{\prime}\right)\right\}, \\
v(g m) & =\chi(g)+v(m), \\
v(r m) & =v(m) \text { when } r \text { is a unit in } R, \\
v(0) & =\infty
\end{aligned}
$$

for all $m, m^{\prime} \in M, g \in G, r \in R$.

The support of $c \in F_{i}$ is the subset $\operatorname{supp}(c)$ of the $R$-basis $G \mathcal{X}_{i}$ consisting of those members which appear with non-zero coefficients in the unique expansion of $c$.

The most important example of a valuation involves extension via supports: Given a free $R G$-module $F$ and an $R G$-basis $\mathcal{X}$ for $F$, every function $v: \mathcal{X} \rightarrow \mathbb{R}$ extends to a unique function $v: G X \rightarrow \mathbb{R} \cup\{\infty\}$ by $v(g x):=\chi(g)+v(x)$, and then to a valuation on $F$ by defining, for each non-zero $c \in F, v(c)=\min \{v(\operatorname{supp}(c))\}$.

Let $\boldsymbol{F} \rightarrow R$ be a free resolution of the trivial $R G$-module $R$. We call this resolution admissible if: (i) each free $R G$-module $F_{i}$ in $\boldsymbol{F}$ comes with a given basis $X_{i}$, and (ii) for each $x \in \mathcal{X}_{i}, \partial x \neq 0 \in F_{i-1}$, while for $x \in \mathcal{X}_{0}, \epsilon(x)=1 \in R$, where $\epsilon: F_{0} \rightarrow R$ denotes the augmentation map. We write $\boldsymbol{F}^{(n)}$ for the $n$-skeleton

\footnotetext{
${ }^{5}$ In general the $F_{n}$ property is stronger than the $\mathrm{FP}_{n}$ property.
} 
$\bigoplus_{i=0}^{n} F_{i}$ which is free with basis $\mathcal{X}^{(n)}=\bigsqcup_{i=0} \mathcal{X}_{i}$. When $G$ has type $\mathrm{FP}_{n}$ there is always an admissible free resolution with finitely generated $n$-skeleton.

If $\boldsymbol{F} \rightarrow R$ is admissible we think of $\boldsymbol{F}$ as $\bigoplus_{i \geq 0} F_{i}$, a free module with basis $\mathcal{X}=$ $\bigsqcup_{i \geq 0} X_{i}$. The basic valuation on $\boldsymbol{F}$ extending $\chi$ is the function $v_{\chi}: \boldsymbol{F} \rightarrow \mathbb{R} \cup\{\infty\}$ of the kind described above, where the values on $\mathcal{X}$ are chosen inductively, skeleton by skeleton, to satisfy

$$
v_{\chi}(x)= \begin{cases}0 & \text { if } i=0, \\ v_{\chi}(\partial x) & \text { if } i>0,\end{cases}
$$

and $v_{\chi}(g x)=\chi(g)+v_{\chi}(x)$ when $g \in G$. It follows that $v_{\chi}(c) \leq v_{\chi}(\partial c)$ for all $c \in \boldsymbol{F}$, and $v_{\chi}(c)=\infty$ if and only if $c=0$.

One shows easily that if $\boldsymbol{F}$ has finitely generated $n$-skeleton then every valuation $v: \boldsymbol{F}^{(n)} \rightarrow \mathbb{R}$ on the $n$-skeleton extending $\chi$ is dominated by the basic valuation $v_{\chi}$ in the sense that there is a number $\mu \geq 0$ with $v(c) \geq v_{\chi}(c)-\mu$ for all $c \in \boldsymbol{F}^{(n)}$.

We note that when $\chi=0$ then $v$ takes all non-zero elements of $\boldsymbol{F}$ to 0 .

\section{The invariant $\Sigma^{n}(G ; R)$}

In this section we recall another definition of $\Sigma^{n}(G ; R)$. Let $\boldsymbol{F} \rightarrow R$ be an admissible free resolution with $\mathcal{X}^{(n)}$ finite. We denote by $\widetilde{\boldsymbol{F}}$ the augmented (exact) chain complex $\boldsymbol{F} \rightarrow R \rightarrow 0$; i.e., $\widetilde{F}_{i}=F_{i}$ for $i \neq-1, \widetilde{F}_{-1}=R$, and we write $\epsilon: F_{0} \rightarrow R$ for the augmentation map. All valuations $v$ on $\boldsymbol{F}$ are extended to $\widetilde{\boldsymbol{F}}$ by the convention $v(r)=\infty$ for every $r \in R$.

If $v$ is a basic valuation extending the non-zero character $\chi$, the property $v(c) \leq$ $v \partial(c)$ ensures that $\widetilde{\boldsymbol{F}}$ carries an $\mathbb{R}$-graded filtration by $R$-subcomplexes $\widetilde{\boldsymbol{F}}_{v}^{[t, \infty]}$ where $t \in \mathbb{R}$ and $\widetilde{F}_{v, i}^{[t, \infty]}:=\left\{c \in \widetilde{F}_{i} \mid v(c) \geq t\right\}$. In particular, $\widetilde{F}_{v,-1}^{[t, \infty]}=R$ for all $t$.

We say that $\widetilde{\boldsymbol{F}}$ is controlled $(n-1)$ - acyclic (abbrev. $\mathrm{CA}^{n-1}$ ) with respect to $v$ if there exists $\lambda \geq 0$ such that, for all $t$ and all $0 \leq p \leq n-1$, the inclusion induces the zero homomorphism

$$
H_{p}\left(\widetilde{\boldsymbol{F}}_{v}^{[t, \infty]}\right) \rightarrow H_{p}\left(\widetilde{\boldsymbol{F}}_{v}^{[t-\lambda, \infty]}\right) .
$$

This condition should be considered vacuous when $n=0$; i.e., $\widetilde{\boldsymbol{F}}$ is always $\mathrm{CA}^{-1}$ with respect to $v$.

Remark 3.1. If the $\mathrm{CA}^{n-1}$-condition holds for some $t$ then $\lambda$ can be chosen so that it holds, using this same $\lambda$, for every $t$. This is because a non-zero character defines a cocompact action on $\mathbb{R}$.

The following is the content of [7], Theorem 3.2. 
Theorem 3.2. Let $\chi: G \rightarrow \mathbb{R}$ be a non-zero character. If $\boldsymbol{F} \rightarrow R$ is an admissible free resolution with finitely generated $n$-skeleton, and $v: \boldsymbol{F} \rightarrow \mathbb{R} \cup\{\infty\}$ is a basic valuation extending $\chi$, then $[\chi] \in \Sigma^{n}(G ; R)$ if and only if $\widetilde{\boldsymbol{F}}$ is $C A^{n-1}$ with respect to $v$.

\section{Valuations on tensor products}

We now consider the product $G \times H$ of two groups. Let $\boldsymbol{F} \rightarrow R$ be an admissible free resolution of the $R G$-module $R$ with basis $X$, and let $\boldsymbol{F}^{\prime} \rightarrow R$ be an admissible free resolution of the $R H$-module $R$ with basis $X^{\prime}$. Then, with respect to the product action, $\boldsymbol{F} \otimes_{R} \boldsymbol{F}^{\prime} \rightarrow R$ is a free resolution, and $\left\{x \otimes x^{\prime} \mid x \in \mathcal{X}, x^{\prime} \in \mathcal{X}^{\prime}\right\}$ is a basis for $\boldsymbol{F} \otimes_{R} \boldsymbol{F}^{\prime}$. Since $\partial\left(x \otimes x^{\prime}\right)=\partial x \otimes x^{\prime} \pm x \otimes \partial x^{\prime}$, simple considerations show that $\boldsymbol{F} \otimes_{R} \boldsymbol{F}^{\prime} \rightarrow R$ is admissible.

Let $\chi: G \rightarrow \mathbb{R}$ and $\chi^{\prime}: H \rightarrow \mathbb{R}$ be characters. Let $v_{\chi}$ and $v_{\chi^{\prime}}^{\prime}$ be the basic valuations on $\boldsymbol{F}$ and $\boldsymbol{F}^{\prime}$ extending $\chi$ and $\chi^{\prime}$ respectively. Denote by $w$ the basic valuation $v_{\left(\chi, \chi^{\prime}\right)}: \boldsymbol{F} \otimes_{R} \boldsymbol{F}^{\prime} \rightarrow \mathbb{R} \cup\{\infty\}$ extending $\left(\chi, \chi^{\prime}\right)$.

Proposition 4.1. $w\left(c \otimes c^{\prime}\right)=v_{\chi}(c)+v_{\chi^{\prime}}^{\prime}(c)$

Proof. We abbreviate $v_{\chi}$ to $v$ and $v_{\chi^{\prime}}^{\prime}$ to $v^{\prime}$. The proof is by induction on the degree $n$ of $w\left(c \otimes c^{\prime}\right)$. The case $n=0$ is easy, so we assume the proposition is true for $n-1$. Let $x \otimes x^{\prime}$ have degree $n \geq 1$. Then

$$
\begin{array}{rlr}
w\left(x \otimes x^{\prime}\right) & =w\left(\partial\left(x \otimes x^{\prime}\right)\right) & \\
& =w\left(\partial x \otimes x^{\prime}+x \otimes \partial x^{\prime}\right) \\
& =\min \left\{w\left(\partial x \otimes x^{\prime}\right), w\left(x \otimes \partial x^{\prime}\right)\right\} & \\
& =\min \left\{v(\partial x)+v^{\prime}\left(x^{\prime}\right), v(x)+v^{\prime}\left(\partial x^{\prime}\right)\right\} & \text { (by induction, since the } \\
& =v(x)+v^{\prime}\left(x^{\prime}\right), &
\end{array}
$$

so

$$
w\left(g x \otimes h x^{\prime}\right)=v(g x)+v^{\prime}\left(h x^{\prime}\right) .
$$

Since $R$ is a domain, $\operatorname{supp}\left(c \otimes c^{\prime}\right)=\operatorname{supp}(c) \times \operatorname{supp}\left(c^{\prime}\right)$. Thus

$$
w\left(c \otimes c^{\prime}\right)=v_{\chi}(c)+v_{\chi^{\prime}}(c) .
$$

\section{Sigma invariants of products}

It is convenient to split the proof of Theorem 1.3 into two propositions.

Proposition 5.1. $[(\chi, 0)] \in \Sigma^{n}(G \times H ; R)$ if and only if $[\chi] \in \Sigma^{n}(G ; R)$. 
Proof. The "if" part of the proposition follows from Meinert's inequality. We are to prove the "only if" part.

With notation as in Section 4 we assume that $\boldsymbol{F}$ and $\boldsymbol{F}^{\prime}$ have finitely generated $n$-skeleta. Then $\boldsymbol{F} \otimes_{R} \boldsymbol{F}^{\prime}$ also has finitely generated $n$-skeleton. Let $v$ denote the basic valuation on $\boldsymbol{F}$ extending the character $\chi$ on $G$. By Proposition 4.1, $w$, defined by $w\left(c \otimes c^{\prime}\right)=v(c)$, is the basic valuation extending the character $(\chi, 0)$ on $G \times H$.

The chain complex $\boldsymbol{F}$ is a retract of $\boldsymbol{F} \otimes_{R} \boldsymbol{F}^{\prime}$ as follows. We may assume (for convenience) that $F_{0}^{\prime}$ is generated by a single generator $x^{\prime}$ and that $x^{\prime}$ is mapped by $\epsilon^{\prime}$ to $1 \in R$. Define $i: \boldsymbol{F} \rightarrow \boldsymbol{F} \otimes_{R} \boldsymbol{F}^{\prime}$ by $i(c)=c \otimes x^{\prime}$. Define $p: \boldsymbol{F} \otimes_{R} \boldsymbol{F}^{\prime} \rightarrow \boldsymbol{F}$ by $p\left(c \otimes c^{\prime}\right)=\epsilon\left(c^{\prime}\right) c$ when $c^{\prime}$ has degree 0 , and $p\left(c \otimes c^{\prime}\right)=0$ when $c^{\prime}$ has degree $>0$. One checks that $i$ and $p$ are chain maps and that $p \circ i$ is the identity map. The composition $v \circ p$ is a valuation on $\boldsymbol{F} \otimes_{R} \boldsymbol{F}^{\prime}$ and is therefore dominated by the basic valuation $w$.

Let $z \in \boldsymbol{F}$ be a $k$-cycle where $k<n$. By assumption there is a number $\lambda \geq 0$ and a $(k+1)$-chain $d \in \boldsymbol{F} \otimes_{R} \boldsymbol{F}^{\prime}$ with $\partial d=i(z)$ and $w(d) \geq w(i(z))-\lambda$. Hence we have

$$
\partial p(d)=p(\partial d)=p(i(z))=z
$$

and

$$
\begin{aligned}
v(p(d)) & =(v \circ p)(d) \\
& \geq w(d)-\mu \\
& \geq w(i(z))-\lambda-\mu \\
& =v(z)-\lambda-\mu .
\end{aligned}
$$

In view of Meinert's Inequality, the new content of Theorem 1.3 is:

Proposition 5.2. Let $R$ be a field. Then

$$
\Sigma^{n}(G \times H ; R)^{c} \supseteq \bigcup_{p=0}^{n} \Sigma^{p}(G ; R)^{c} * \Sigma^{n-p}(H ; R)^{c} .
$$

Proof. The cases $p=0$ and $p=n$ (mutatis mutandis) are covered by Proposition 5.1, so we will assume $1 \leq p \leq n-1$. As before, $v$ denotes a basic valuation on $\boldsymbol{F}$ extending the character $\chi$ on $G$, and $v^{\prime}$ denotes a basic valuation on $\boldsymbol{F}^{\prime}$ extending the character $\chi^{\prime}$ on $H$. We are to show that if $[\chi] \in \Sigma^{p}(G ; R)^{c}$ and $\left[\chi^{\prime}\right] \in \Sigma^{n-p}(H ; R)^{c}$ then $\left[\left(\chi, \chi^{\prime}\right)\right] \in \Sigma^{n}(G \times H ; R)^{c}$.

We view $G \mathcal{X} \times H \mathcal{X}^{\prime}$ as the $R$-basis of $\boldsymbol{F} \otimes_{R} \boldsymbol{F}^{\prime}$. We denote by $p$ and $p^{\prime}$ the projections onto the two factors $G \mathcal{X}$ and $H \mathcal{X}^{\prime}$. Given $u \in \mathbb{R}$, each chain $y \in \boldsymbol{F} \otimes_{R} \boldsymbol{F}^{\prime}$ has a unique decomposition $y=y_{\lambda}+y_{\rho}$ where

$$
v\left(p\left(\operatorname{supp}\left(y_{\lambda}\right)\right)\right)<u \leq v\left(p\left(\operatorname{supp}\left(y_{\rho}\right)\right)\right) .
$$

Thus, $y_{\rho}$ is the "subchain" obtained from $y$ by setting equal to zero the coefficients of all basis elements $g x \otimes h x^{\prime}$ such that $g x$ does not lie in $\boldsymbol{F}_{v}^{[u, \infty]}$. (We think of $\lambda$ and $\rho$ as standing for "left" and "right" - see Figure 2.) We observe: 
(1) For all $y \in \boldsymbol{F} \otimes_{R} \boldsymbol{F}^{\prime}, y_{\lambda}=0$ if and only if $v(p(\operatorname{supp}(y))) \geq u$.

(2) Thus, in particular, $\left(c \otimes c^{\prime}\right)_{\lambda}=0$ if and only if $v(c)=0$.

(3) If $d$ and $e$ in $\boldsymbol{F} \otimes_{R} \boldsymbol{F}^{\prime}$ have disjoint supports then $(d+e)_{\lambda}=d_{\lambda}+e_{\lambda}$.

Similarly, given $u^{\prime} \in \mathbb{R}$, each chain $y$ has a unique decomposition $y=y_{\beta}+y_{\tau}$ where

$$
v^{\prime}\left(p^{\prime}\left(\operatorname{supp}\left(y_{\beta}\right)\right)\right)<u^{\prime} \leq v^{\prime}\left(p^{\prime}\left(\operatorname{supp}\left(y_{\beta}\right)\right)\right) .
$$

Thus, $y_{\tau}$ is the "subchain" obtained from $y$ by setting equal to zero the coefficients of all basis elements $g x \otimes h x^{\prime}$ such that $h x^{\prime}$ does not lie in $\boldsymbol{F}_{v}^{\prime\left[u^{\prime}, \infty\right]}$. (We think of $\beta$ and $\tau$ as standing for "bottom" and "top".) We call the (fixed) numbers $u$ and $u^{\prime}$ splitters; they must be specified before this notation can be used.

Without loss of generality we may assume $\widetilde{\boldsymbol{F}}$ is $\mathrm{CA}^{p-2}$ with respect to $v$ and $\widetilde{\boldsymbol{F}}^{\prime}$ is $\mathrm{CA}^{n-p-2}$ with respect to $v^{\prime}$. For a chosen $(p-1)$-cycle $z \in \boldsymbol{F}$ and a chosen $(n-p-1)$-cycle $z^{\prime} \in \boldsymbol{F}^{\prime}$ define

$$
\eta(z):=\inf \{v(z)-v(c) \mid \partial c=z\}
$$

and

$$
\eta^{\prime}\left(z^{\prime}\right):=\inf \left\{v^{\prime}\left(z^{\prime}\right)-v^{\prime}\left(c^{\prime}\right) \mid \partial c^{\prime}=z^{\prime}\right\} .
$$

The numbers $\eta(z)$ and $\eta^{\prime}\left(z^{\prime}\right)$ can be made arbitrarily large by suitable choice of $z$ and $z^{\prime}$. Once $z$ and $z^{\prime}$ have been chosen, we choose positive numbers $\mu<\eta(z)$ and $\mu^{\prime}<\eta^{\prime}\left(z^{\prime}\right)$. Then choose chains $c$ and $c^{\prime}$ such that $\partial c=z, \partial c^{\prime}=z^{\prime}, v(z)-\mu-1<$ $v(c) \leq v(z)-\mu$ and $v^{\prime}\left(z^{\prime}\right)-\mu^{\prime}-1<v^{\prime}\left(c^{\prime}\right) \leq v^{\prime}\left(z^{\prime}\right)-\mu^{\prime}$. See Figure 1 .

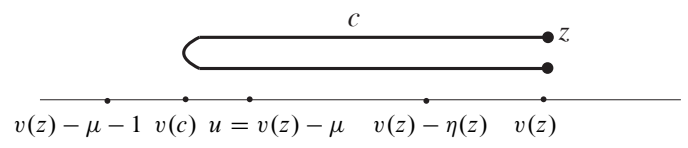

Figure 1.

Since $c \otimes c^{\prime}$ is an $n$-chain, and $\partial\left(c \otimes c^{\prime}\right)=z \otimes c^{\prime} \pm c \otimes z^{\prime}$ we have

$$
\begin{aligned}
w\left(\partial\left(c \otimes c^{\prime}\right)\right) & =w\left(z \otimes c^{\prime} \pm c \otimes z^{\prime}\right) \\
& \geq \min \left\{w\left(z \otimes c^{\prime}\right), w\left(c \otimes z^{\prime}\right)\right\} \\
& =\min \left\{v(z)+v^{\prime}\left(c^{\prime}\right), v(c)+v^{\prime}\left(z^{\prime}\right)\right\} \\
& >\min \left\{v(z)+v^{\prime}\left(z^{\prime}\right)-\mu^{\prime}-1, v(z)+v^{\prime}\left(z^{\prime}\right)-\mu-1\right\} \\
& =w\left(z \otimes z^{\prime}\right)-1-\max \left\{\mu, \mu^{\prime}\right\} .
\end{aligned}
$$

Now we take as our splitters $u:=v(z)-\mu$ and $u^{\prime}:=v^{\prime}\left(z^{\prime}\right)-\mu^{\prime}$. We consider an arbitrary $n$-chain $d$ such that $\partial d=\partial\left(c \otimes c^{\prime}\right)$. Define

$$
b:=\partial\left(d_{\lambda}\right)-(\partial d)_{\lambda}
$$


and define $e:=\partial\left(b_{\beta}\right)$. Thus $e$ is an $(n-2)$-cycle. See Figure 2.

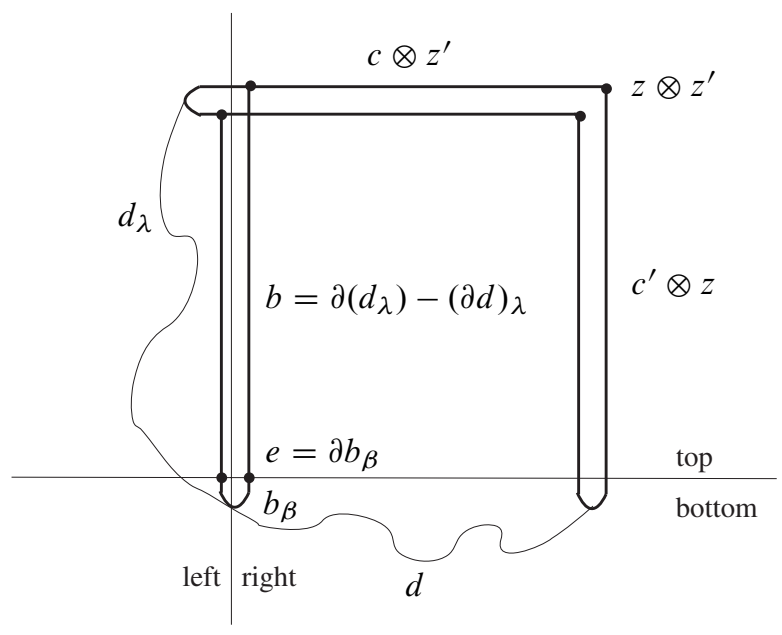

Figure 2.

Claim 1: $(\partial d)_{\lambda}= \pm\left(c \otimes z^{\prime}\right)_{\lambda}$.

Proof. $\partial d=\partial\left(c \otimes c^{\prime}\right)=z \otimes c^{\prime} \pm c \otimes z^{\prime}$. Since $v\left(p\left(\operatorname{supp}\left(z \otimes c^{\prime}\right)\right)\right) \geq$ $v(\operatorname{supp}(z))>u$ we have $\left(z \otimes c^{\prime}\right)_{\lambda}=0$. Thus

$$
(\partial d)_{\lambda}=\left(z \otimes c^{\prime}\right)_{\lambda} \pm\left(c \otimes z^{\prime}\right)_{\lambda}= \pm\left(c \otimes z^{\prime}\right)_{\lambda},
$$

as claimed.

Claim 1 is used in

$$
\begin{aligned}
z \otimes z^{\prime} & =\partial\left(c \otimes z^{\prime}\right) \\
& =\partial\left(\left(c \otimes z^{\prime}\right)_{\lambda}\right)+\partial\left(\left(c \otimes z^{\prime}\right)_{\rho}\right) \\
& = \pm \partial\left((\partial d)_{\lambda}\right)+\partial\left(\left(c \otimes z^{\prime}\right)_{\rho}\right) \\
& = \pm \partial(b)+\partial\left(\left(c \otimes z^{\prime}\right)_{\rho}\right) \\
& = \pm \partial\left(b_{\beta}\right) \pm \partial\left(b_{\tau}\right)+\partial\left(\left(c \otimes z^{\prime}\right)_{\rho}\right) \\
& = \pm e \pm \partial\left(b_{\tau}\right)+\partial\left(\left(c \otimes z^{\prime}\right)_{\rho}\right) .
\end{aligned}
$$

Next we show that the indicated homology between $z \otimes z^{\prime}$ and $e$ takes place in the chain complex

$$
\boldsymbol{C}:=\boldsymbol{F}_{v}^{[u, \infty]} \otimes \boldsymbol{F}_{v^{\prime}}^{\prime\left[u^{\prime}, \infty\right]}
$$

Claim 2: $b=\left(\partial\left(d_{\lambda}\right)\right)_{\rho}$.

Proof. $d=d_{\lambda}+d_{\rho}$. So $\partial d=\partial\left(d_{\lambda}\right)+\partial\left(d_{\rho}\right)$. Thus $(\partial d)_{\lambda}=\left(\partial\left(d_{\lambda}\right)\right)_{\lambda}$, because $\boldsymbol{F}_{v}^{[u, \infty]}$ is a chain complex, so $\left(\partial\left(d_{\rho}\right)\right)_{\lambda}=0$. Since $b=\left(\partial\left(d_{\lambda}\right)\right)_{\rho}+\left(\partial\left(d_{\lambda}\right)\right)_{\lambda}-(\partial d)_{\lambda}$ the claim follows. 
Claim 3: $c \otimes z^{\prime}=\left(c \otimes z^{\prime}\right)_{\tau}$.

Proof. This is because $v^{\prime} p^{\prime}\left(\operatorname{supp}\left(c \otimes z^{\prime}\right)\right)>u^{\prime}$.

It follows from Claims 2 and 3 that $b_{\tau}=\left(b_{\rho}\right)_{\tau}$ and $\left(c \otimes z^{\prime}\right)_{\rho}=\left(\left(c \otimes z^{\prime}\right)_{\tau}\right)_{\rho}$, so $e$ and $z \otimes z^{\prime}$ are homologous in $\boldsymbol{C}$.

We now use the fact that $z$ does not bound in $\widetilde{\boldsymbol{F}}_{v}^{[u, \infty]}$ and $z^{\prime}$ does not bound in $\widetilde{\boldsymbol{F}}^{\prime}{ }_{v^{\prime}}^{\left[u^{\prime}, \infty\right]}$. Because $R$ is a field, the Künneth Formula ([16], Lemma 5.3.1) applied to $\boldsymbol{C}$ implies that the homology class of $z \otimes z^{\prime}$ in $C$ could only be zero if the homology class of either $z$ or $z^{\prime}$ is zero in the appropriate tensor factor of $\boldsymbol{C}$; and neither is zero, as we have just seen. Thus the homology class of $e$ is non-zero, and hence the cycle $e=\partial\left(b_{\beta}\right)$ is non-zero. It follows that $b_{\beta}$ is non-zero. Then Claim 2 implies that $\left(\partial\left(d_{\lambda}\right)_{\rho}\right)_{\beta}$ is non-zero.

Claim 4: $\left(d_{\lambda}\right)_{\beta} \neq 0$.

Proof. Since the support of $\left(\partial\left(d_{\lambda}\right)_{\rho}\right)_{\beta}$ is non-zero, the support of $\left(\partial\left(d_{\lambda}\right)_{\rho}\right)$ contains some $g x \otimes h x^{\prime}$ with $v^{\prime}\left(h x^{\prime}\right)<u^{\prime}$. So the same is true of $\partial\left(d_{\lambda}\right)$. Hence the support of $d_{\lambda}$ contains some $\tilde{g} \tilde{x} \otimes \tilde{h} \tilde{x}^{\prime}$ with $v^{\prime}\left(\tilde{h} \tilde{x}^{\prime}\right)<u^{\prime}$. Thus $\left(d_{\lambda}\right)_{\beta} \neq 0$, as claimed.

By Claim 4, we get

$$
\begin{aligned}
w(d) & \leq w\left(\tilde{g} \tilde{x} \otimes \tilde{h} \tilde{x^{\prime}}\right) \\
& =v(\tilde{g} \tilde{x})+v^{\prime}\left(\tilde{h} \tilde{x}^{\prime}\right) \\
& \leq v(z)-\mu+v^{\prime}\left(z^{\prime}\right)-\mu^{\prime} \\
& =w\left(z \otimes z^{\prime}\right)-\mu-\mu^{\prime} .
\end{aligned}
$$

Summarizing, since $\partial d=\partial\left(c \otimes c^{\prime}\right)$ we conclude that

$$
w(\partial d)-w(d) \geq w\left(z \otimes z^{\prime}\right)-1-\max \left\{\mu, \mu^{\prime}\right\}-w\left(z \otimes z^{\prime}\right)+\mu+\mu^{\prime},
$$

i.e.,

$$
w(\partial d)-w(d) \geq \min \left\{\mu, \mu^{\prime}\right\} .
$$

Since $\mu$ and $\mu^{\prime}$ can be chosen arbitrarily large, say $>N$, the cycle $\partial\left(c \otimes c^{\prime}\right)$ has the property that for any $d$ with $\partial d=\partial\left(c \otimes c^{\prime}\right), w(\partial d)-w(d)>N-1$. In other words,

$$
\widetilde{\boldsymbol{F} \otimes_{R} \boldsymbol{F}^{\prime}} \text { is not } \mathrm{CA}^{n-1} \text { with respect to } w \text {. }
$$

Remark 5.3. The only place where we needed $R$ to be a field was to ensure that (referring to homology classes) $\left\{z \otimes z^{\prime}\right\}=0$ forces $\{z\}=0$ or $\left\{z^{\prime}\right\}=0$. This also holds when $R=\mathbb{Z}$ provided $\{z\}$ and $\left\{z^{\prime}\right\}$ have infinite order. Thus our proof also gives Theorem 1.4 because under the hypotheses of Theorem 1.4 the $\mathbb{Z}$-cycles $z$ and $z^{\prime}$ can always be chosen so that their homology classes have infinite order. (This is just a variant on the proof given in Section 1.) We also get Theorem 1.5 because in the cases where both sides of the join are non-empty the dimensions of the relevant cycles are 0 and 1 , and the 0 -cycle can always be chosen to be indivisible. 


\section{References}

[1] M. Bestvina and N. Brady, Morse theory and finiteness properties of groups. Invent. Math. 129 (1997), 445-470. Zbl 0888.20021 MR 1465330

[2] R. Bieri, Finiteness length and connectivity length for groups. In Geometric group theory down under (Canberra, 1996), de Gruyter, Berlin 1999, 9-22. Zbl 1009.20048 MR 1714837 252, 253, 254

[3] R. Bieri and R. Geoghegan, Connectivity properties of group actions on non-positively curved spaces. Mem. Amer. Math. Soc. 161 (2003), no. 765.Zbl 1109.20035 MR 1950396

[4] R. Bieri and R. Geoghegan, Paper in prepartion.

[5] R. Bieri, R. Geoghegan, and D. Kochloukova, The Sigma invariants of Thompson's group F. Groups Geom. Dyn. 4 (2010), 263-273. 251, 254

[6] R. Bieri, W. D. Neumann, and R. Strebel, A geometric invariant of discrete groups. Invent. Math. 90 (1987), 451-477. Zbl 0642.57002 MR 914846252

[7] R. Bieri and B. Renz, Valuations on free resolutions and higher geometric invariants of groups. Comment. Math. Helv. 63 (1988), 464-497. Zbl 0654.20029 MR 960770 251, 252,255

[8] K.-U. Bux and C. Gonzalez, The Bestvina-Brady construction revisited: geometric computation of $\Sigma$-invariants for right-angled Artin groups. J. London Math. Soc. (2) 60 (1999), 793-801. Zbl 1025.20026 MR 1753814 253

[9] R. Gehrke, Die höheren geometrischen Varianten für Gruppen mit Kommutatorrelationen. Dissertation, University of Frankfurt am Main, 1992. 254

[10] R. Gehrke, The higher geometric invariants for groups with sufficient commutativity. Comm. Algebra 26 (1998), 1097-1115. Zbl 0905.20024 MR 1612192 253, 254

[11] R. Geoghegan, Topological methods in group theory. Grad. Texts in Math. 243, Springer, New York 2008. Zbl 1141.57001 MR 2365352 252

[12] J. Meier, H. Meinert, and L. VanWyk, On the $\Sigma$-invariants of Artin groups. Topology Appl. 110 (2001), 71-81. Zbl 1037.20037 MR 1804699 253

[13] J. Meier, H. Meinert, and L. VanWyk, On the $\Sigma$-invariants of graph products based on trees. Preprint. 252

[14] H. Meinert, Über die höheren geometrischen Invarianten für endliche direkte Produkte von Gruppen. Diploma thesis, University of Frankfurt am Main, 1990. 254

[15] D. Schütz, On the direct product conjecture for sigma invariants. Bull. Lond. Math. Soc. 40 (2008), 675-684. Zbl 05316994 MR 2441140 253, 254

[16] E. H. Spanier, Algebraic topology. Springer-Verlag, New York 1981. Zbl 0477.55001 MR 0666554260

Received April 15, 2009; revised June 15, 2009

R. Bieri, FB 12 - Institut für Mathematik, Johann Wolfgang Goethe-Universität Frankfurt, Robert-Mayer-Str. 6-8, 60325 Frankfurt am Main, Germany

E-mail: bieri@math.uni-frankfurt.de 
R. Geoghegan, Department of Mathematical Sciences, Binghamton University (SUNY), Binghamton, NY 13902-6000, U.S.A.

E-mail: ross@math.binghamton.edu 